\title{
Tripartite Evolutionary Game Analysis on Selection Behavior of Trans-Regional Hospitals and Patients in Telemedicine System
}

\author{
Yuxuan Gao, Yueping Du, Bingzhen Sun, Rui Wang, Chaoying Jiang \\ School of Economics and Management, Xidian University, \\ Xi'an, Shaanxi, China \\ E-mail: bzsun@xidian.edu.cn; gaoyuxuan@xidian.edu.cn.
}

Received 27 November 2016

Accepted 29 June 2017

\begin{abstract}
This study applies the game theory to the discussion and analysis of trans-regional Telemedicine System, builds the game model of the selection strategies of trans-regional hospitals and patients and analyzes evolving paths, equilibrium states and influencing factors of the three parties. It is derived that medical insurance reimbursement proportion of specialized hospitals, government support for general hospitals and medical expenses in specialized hospitals, operating costs of general hospitals are the influential factors in the Telemedicine System. Finally, a numerical stimulation is conducted with Matlapb based on the data from China Health and Family Planning Statistical Yearbook 2015.
\end{abstract}

Keywords: telemedicine system; medical services; general hospitals; specialized hospitals; patients; evolutionary game.

\section{Introduction}

China's economy has developed since the reform and opening up. The development of network technology brings tremendous changes. People in different regions and in different languages can communicate on the Internet and enjoy trans-regional instantaneous services. For example, in various network communities and on e-commerce platforms. People enjoy the conveniences from science and technology by free communications and easy shopping, and make seriatim comparisons of various brands indoors. Furthermore, by social apps like Wechat and QQ, only a few flow and fees can enable video conversations with relatives and friends abroad which are beyond traditional means of communications.

Though it is easy to achieve distant real-time communication and service sharing theoretically and technically, the development of technology and economy merely improves our living standards and has little influence on can culture and medical treatment for natural conditions and other objective factors. The unique natural conditions, vest territory, the uneven distribution of population and great economy gap between urban and rural areas cause the extremely imbalanced distribution of medical resources. Developed areas have high-quality medical services, while remote areas suffer poor medical conditions and low-level services. For example, the unique geographical environment (high hills and canyons) in Derung Reiver village, Gongshan county, Nujiang prefecture, Yunnan province isolated the people of Derung from the outside, and heavy snow continues half a year. In the end of 2014, the Delung River highway tunnel in the Mount Gaoligong ended the isolated life of Derung people, and pushed a direct transition from primitive society to modern society. Transportation services bring a 
lot of conveniences and benefits to Derung people, mainly in freight transportation and personnel exchanges. When it comes to medical services, the traditional ideas, languages and living habits because of long-time isolation hinder them to go for medical services in highly modernized hospitals. Therefore, they prefer to see doctors locally. So it is urgent for those remote mountain areas to introduce medical services in developed areas. And it is worthy of further study for the state and government departments to develop appropriate policies to ensure that the masses can get the same medical services.

Telemedicine services, an emerging technology in the field of Internet and medicine, are a high-end science and technology and integrate computer information management system, modern communications and modern medicine ( $\mathrm{Fu} 2006)$. It can enable patients in remote areas to enjoy high-quality medical services, reduce medical expenses, improve medical efficiency to avoid the delay of treatment, and greatly improve the primary healthcare services. As a result, telemedicine services have made it possible for people in remote mountain areas to get the same high-quality medical services as those in the developed areas without additional charges or high costs. At present, unified planning and deployment is needed to promote the continuous development of telemedicine services. Therefore, the state and government departments should pay high priority to formulate policies to expand the development of telemedicine services.

In view of existing issues and current situations on trans-regional collaboration and the choices of patients in the study of telemedicine services, and the superiority of the game theory in decision making under uncertainty. It is innovative to use evolutionary game theory in the field of telemedicine. This paper first consider the tripartite groups in telemedicine services, introduces the game theory into the telemedicine services problems, and then established the evolutionary model of the selection behavior of general hospitals, specialist hospitals and patients in combination with Chinas actual conditions, on the basis of the existing research (Zheng et al. 2013), analyzed the evolving paths, equilibrium states and related indexes and studied the factors in- fluencing the development of telemedicine services on the principle of maximizing the three parties' profit. In order to more close to the actual situation of China, real data of the book China Health and Family Planning Statistical Yearbook 2015 is for the first time used for numerical analysis. China Health and Family Planning Statistical Yearbook is an informative annuals reflecting the family planning career development in China's health and the health of the residents. Due to reflect all of China's medical industry in the yearbook detail data, in order to more close to the real China, we are according to the need to filter some trans-regional indicators in this paper, make a conclusion through numerical test with matlab. Finally, the corresponding incentive policies and recommendations are given. This paper use recent real data and the results are of reference value to develop China's telemedicine services and even to reform China's healthcare system. It should be pointed out that due to the limitations of data statistics, it is only applicable for the data to the areas where has a big gap in economy and health. After the data take into, it does not reflect the difference for the city where the level of economic development is much the same.

The rest of this paper is organized as follows. In Section 2, we first review the literature about telemedicine services and game theory. In Section 3 , we briefly the problems of telemedicine services and then set the parameters. In Section 4, we establish and solve the evolutionary model of the selection behavior of general hospitals, specialist hospitals and patients. In Section 5, we applies our approach through a case study adopting real data of the book China Health and Family Planning Statistical Yearbook 2015. At last, we conclude our research and set out further research directions in Section 6.

\section{Literature review}

Telemedicine System has become the focus of scholars at home and abroad, but the selection behavior of trans-religion hospitals and patients in Telemedicine system requires further studies. For example, Wang (2012) analyzed the reasons restricting the development of telemedicine services from the aspects of 
concepts, policies, regulations and standards, and proposed his suggestions in combination with the actual conditions in our country. Rajan et al. (2013) analyzed the influence of introducing telemedicine technology on the choice of patients, and discussed the resulting changes of market shares of specialized and community hospitals through the establishment of mathematical models in detail. Zanaboni (2012) pointed out the factors influencing the promotion and development of telemedicine technology, and proposed the appropriate incentive measures by analyzing the development of telemedicine status. Ye (2006) refined the functional model of telemedicine system, and then proposed a reference model to support telemedicine system architecture , discussed the enabling technology to support collaborative diagnosis and treatment through analyzing the composition principle of telemedicine system and the provided services. Wang (2014) established a fair remote retrieval (FRR) model to examine and retrieve remote data to protect encrypted documents and data, which can addresses the data security problems of intelligent medical cloud computing. Gan et al. (2014) analyzed medical service system composed of hospitals, health insurance funds and patients, and studied the relationship of medical insurance reimbursement proportion, selection behavior of patients and medical expenses. Ai (2015) conducted an evolutionary analysis of the choice of hospitals and patients for medical APPs, and suggested to guide hospitals to provide medical APPs for patients to choose. Sarah (2017) developed a decision support tool for multi-skill health care tasks scheduling in the Pediatric Emergency Department.

Game theory, as a new mathematical theory and tool to handle with complexity decision making problem under uncertainty, was proposed by Von Neumann and Morgenstern (1944). In the past years, game theory has become the core of content in the mainstream in the economics and management field. Moreover, it has almost become the basic analytical tools and study language of economists and management experts in the entire field. So far it has successfully applied to many areas such as optimization problem (Jin 2010), matrix games ( $\mathrm{Li}$ et al. 2015a, Nan et al.2013, Tina et al. 2015), cooper- ative games (Xie et al. 2015), fuzzy information( $\mathrm{Li}$ et al. 2015b, 2015c), group decision(Li 2014), multiattribute decision(Li and Wan 2013), fuzzy games (Meng et al. 2013; Meng 2015; An et al. 2016), fuzzy multichoice games (Meng et al. 2016), environmental protection (Zhao et al. 2015, 2016). Its application combined with other uncertainty mathematical theories (Sun et al. 2014; 2015a,b; 2016a) , rough set theory (Sun et al.2016b,c; 2017), algorithm innovation (Gu et al. 2016a,b; Zheng et al. 2015; Wen et al. 2015), collaborative recommender (Ma et al. 2015), linear programming problem (Zhang et al. 2016) and global context (Zhou et al. 2017).

Most of the existing researches provide systematical designs from the technical level or evolve from specific medical media. This provides a practical background for the evolutionary game analysis of related groups in telemedicine services and enriches the related theories. But little consideration has been given to the evolutionary analysis of the selection behavior of related groups, and the related conclusions and suggestions on promoting the development of telemedicine services are not enough. In addition, most of the existing studies about evolutionary game theory use random generated experimental data for numerical simulation. In reality, due to the large differences between random experimental data and real data, it is difficult to truly analyze the existing problems in China. However, these problems just need careful consideration to reform China's medical System and improve the medical service system.

Considering the selection behavior among general hospitals in the area where the medical resources are scarce, specialist hospitals where the medical resources are rich and patients. The relationship between general hospitals and specialist hospitals is still essentially competitive, although they have cooperative behavior. And the patient is not completely rational. They all want to pay a minimum cost and obtain maximum benefits. It is the most suitable tool for game theory to solve this problem. This paper intends to study this problem by adopting evolutionary game theory. Only a few people have done research about this problem, but it is 
only a preliminary study. Wang (2015) consider the selection behavior of basic-level hospitals between patients, while ignoring the most important subject participation of the telemedicine system.

\section{Problem Statement and Assumption}

\subsection{Problem Statement}

In the game theory of trans-regional hospitals and patients, telemedicine system consists of specialized hospitals in developed areas, general hospitals in remote areas, and patients. In reality, patients primarily tend to local general hospitals. But if the hospitals failed to provide proper treatment, they could send an invitation to specialized hospitals for remote medical services. (Usually, specialized hospitals provide better-effective treatment than general hospitals.) Specialized hospitals enjoy the rights to accept the invitation or not. In this context, patients reserve their rights to take the telemedicine services or to turn to specialized hospitals on their own. Figure 1 illustrates the game theory of trans-regional hospitals and patients.

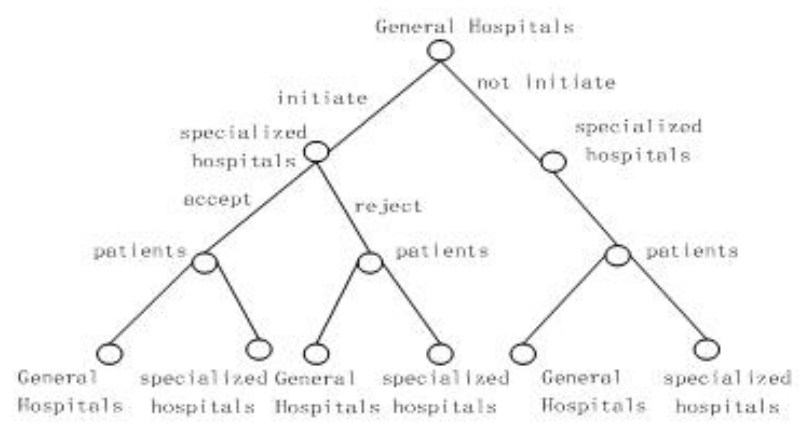

Fig. 1. Game theory of trans-regional hospitals and patients

\subsection{Assumption}

Now, take a look at variable descriptions in the game theory of trans-regional hospitals and patients.Assume that $x \quad(0 \leqslant x \leqslant 1)$ is the probability for general hospitals sending an invitation to specialized hospitals. $E_{1}$ specifies clinical cost of general hospitals, $M_{1}$ specifies government fund for remote cooperative medical services, $C_{1}$ specifies operation cost of general hospitals, and $C_{2}$ specifies their cooperation cost with specialized hospitals. Suppose that government only provides policy support, then $M_{1}<C_{1}$.

Assume that $y(0 \leqslant y \leqslant 1)$ is the probability for specialized hospitals accepting an invitation from general hospitals. $E_{2}$ specifies clinical cost of specialized hospitals, $M_{2}$ specifies government fund for remote cooperative medical services. Suppose that $C_{2}$ specifies the income of specialized hospitals if patients accept medical services at general hospitals. But if patients accept medical services at specialized hospitals, the factual income of specialized hospitals is displayed as $E_{2}+C_{2}$, their operation cost $C_{3}$.

Assume that $z(0 \leqslant z \leqslant 1)$ is the probability for patients to accept medical services at general hospitals. $P_{1}$ specifies the cost of patients, $\mu_{1} \quad\left(0<\mu_{1}<\right.$ 1) specifies the radio of medical insurance, and the factual cost of patients is $\left(1-\mu_{1}\right) P_{1}$.

As for treatment effects, suppose that $V_{1}$ specifies the treatment effects of patients when specialized hospitals lack cooperation with general hospitals. Generally, patients fail to get a fine treatment if there is no cooperation between general hospitals and specialized hospitals. In consequence, patients express their dissatisfaction with certain treatments, then it is concluded that $V_{2}>\left(1-\mu_{1}\right) P_{1}$. On the other side, suppose that $V_{2}$ specifies the treatment effects of patients when specialized hospitals cooperate with general hospitals. Actually, it is not guaranteed that the treatment effects in this case could be the same as the treatment effects provided by specialized hospitals. However, it is known that $V_{2}>V_{1}$, and the satisfaction of patients is $V_{2}>$ $\left(1-\mu_{1}\right) P_{1}$.

As for treatment cost, suppose that $P_{2}$ specifies the cost (including transportation, accommodation and treating fee paid for medicine specialist) for medical services at specialized hospitals, then $P_{2}>P_{1}$. Since the radio of medical insurance is $\mu_{2}$ $\left(0<\mu_{2}<1\right)$ (in reality, the radio of medical insurance of trans-regional hospitals is lower than that of local hospitals, then $\mu_{2}>\mu_{1}$ ), the factual cost of patients is $\left(1-\mu_{2}\right) P_{2}$, the effect they acquired is $V_{3}\left(V_{3} \geqslant V_{2}>V_{1}\right)$. Patients may dissatisfied with the actual treatment effect due to the extra fees paid 
for specialized hospitals, then it is concluded that $V_{3}<\left(1-\mu_{2}\right) P_{2}$.

\section{The Modeling}

This paper analyzes the process of trans-regional hospitals and patients by applying "dynamic game with complete information" theory. In order to derive the revenue function of each side, this paper illustrates the revenue matrix in Figure 2.

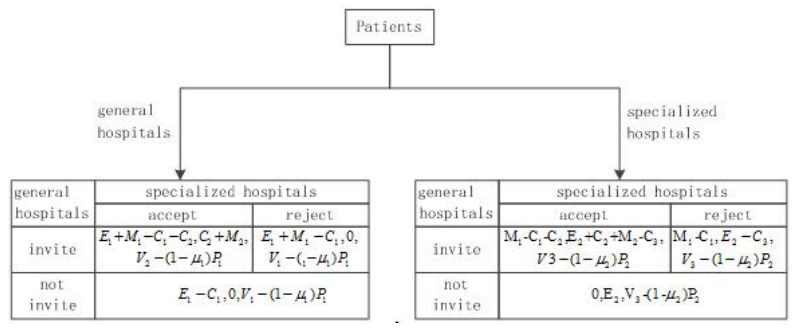

Fig. 2. Revenue matrix of general hospitals, specialized hospitals and patients

The equation of treatment effect that patients received at general hospitals is displayed as follow:

$$
\begin{aligned}
U_{z} & =x y\left(V_{2}-\left(1-\mu_{1}\right) P_{1}\right)+x(1-y)\left(V_{1}-\right. \\
& \left.\left(1-\mu_{1}\right) P_{1}\right)+(1-x)\left(V_{1}-\left(1-\mu_{1}\right) P_{1}\right) \\
& =x y\left(V_{2}-V_{1}\right)+V_{1}-\left(1-\mu_{1}\right) P_{1}
\end{aligned}
$$

The equation of treatment effect that patients received at specialized hospitals is displayed as follow:

$$
\begin{aligned}
U_{(1-z)} & =x y\left(V_{3}-\left(1-\mu_{2}\right) P_{2}\right)+x(1-y)\left(V_{3}-\right. \\
& \left.\left(1-\mu_{2}\right) P_{2}\right)+(1-x)\left(V_{3}-\left(1-\mu_{2}\right) P_{2}\right) \\
& =V_{3}-\left(1-\mu_{2}\right) P_{2}
\end{aligned}
$$

The equation of average treatment effect is displayed as follow:

$$
\bar{U}_{z, 1-z}=z U_{z}+(1-z) U_{1-z}
$$

The replicator dynamics equation of patients is displayed as follow:

$$
\begin{aligned}
F(z) & =\frac{d z}{d t}=z\left(U_{z}-\bar{U}_{z, 1-z}\right) \\
& =z\left(U_{z}-\left(z U_{z}+(1-z) U_{1-z}\right)\right) \\
& =z(1-z)\left(U_{z}-U_{1-z}\right)
\end{aligned}
$$

Combine the first tow equations to the third one, it is derived that:

$$
\begin{aligned}
F(z)=\frac{d z}{d t}= & z(1-z)\left(x y\left(V_{2}-V_{1}\right)+V_{1}-\right. \\
& \left.\left(1-\mu_{1}\right) P_{1}-V_{3}+\left(1-\mu_{2}\right) P_{2}\right)
\end{aligned}
$$

Likewise, the replicator dynamics equations of general hospitals and specialized hospitals are:

$$
\begin{aligned}
& F(x)=\frac{d x}{d t}=x(1-x)\left(M_{1}-C_{1}+z C_{1}-y C_{2}\right) \\
& F(y)=\frac{d y}{d t}=y(1-y)\left(x E_{2}+x C_{2}+x M_{2}-x C_{3}-x z E_{2}\right. \\
& \left.+x z C_{3}+z E_{2}-z C_{3}-E_{2}+C_{3}\right) \\
& \left\{\begin{aligned}
F(x)=\frac{d x}{d t}= & x(1-x)\left(M_{1}-C_{1}+z C_{1}-y C_{2}\right) \\
F(y)=\frac{d y}{d t}= & y(1-y)\left(x E_{2}+x C_{2}+x M_{2}-x C_{3}\right. \\
& \left.-x z E_{2}+x z C_{3}+z E_{2}-z C_{3}-E_{2}+C_{3}\right) \\
F(z)=\frac{d z}{d t}= & z(1-z)\left(x y\left(V_{2}-V_{1}\right)+V_{1}-\left(1-\mu_{1}\right) P_{1}\right. \\
& \left.-V_{3}+\left(1-\mu_{2}\right) P_{2}\right)
\end{aligned}\right.
\end{aligned}
$$

$$
\begin{gathered}
J=\left[\begin{array}{ccc}
a_{1} & a_{2} & a_{3} \\
b_{1} & b_{2} & b_{3} \\
c_{1} & c_{2} & c_{3}
\end{array}\right]=\left[\begin{array}{ccc}
\frac{\partial F(x)}{\partial x} & \frac{\partial F(x)}{\partial y} & \frac{\partial F(x)}{\partial z} \\
\frac{\partial F(y)}{\partial x} & \frac{\partial F(y)}{\partial y} & \frac{\partial F(y)}{\partial z} \\
\frac{\partial F(z)}{\partial x} & \frac{\partial F(z)}{\partial y} & \frac{\partial F(z)}{\partial z}
\end{array}\right] \\
=\left[\begin{array}{c}
(1-2 x)\left(M_{1}-C_{1}+z C_{1}-y C_{2}\right) \\
y(1-y)\left(E_{2}+C_{2}+M_{2}-C_{3}-z E_{2}+z C_{3}\right) \\
y z(1-z)\left(V_{2}-V_{1}\right) \\
-x(1-x) C_{2} \\
(1-2 y)\left(x E_{2}+x C_{2}+x M_{2}-x C_{3}-x z E_{2}+x z C_{3}+z E_{2}-z C_{3}-E_{2}+C_{3}\right) \\
y z(1-z)\left(V_{2}-V_{1}\right) \\
x(1-x) C_{1} \\
y(1-y)\left(E_{2}-C_{3}-x E_{2}+x C_{3}\right) \\
(1-2 z)\left[x y\left(V_{2}-V_{1}\right)+V_{1}-\left(1-\mu_{1}\right) P_{1}-V_{3}+\left(1-\mu_{2}\right) P_{2}\right]
\end{array}\right]
\end{gathered}
$$

For $F(x)=0, x^{*}=0$ or $x^{*}=1$ or $x_{1}^{*}=$ $\frac{D+\sqrt{D^{2}-4 A B}}{2 A}$ or $x_{2}^{*}=\frac{D-\sqrt{D^{2}-4 A B}}{2 A}$

For $F(y)=0, y^{*}=0$ or $y^{*}=1$ or $y_{1}^{*}=$ $\frac{2 A F}{D+\sqrt{D^{2}-4 A B}}$ or $y_{2}^{*}=\frac{2 A F}{D-\sqrt{D^{2}-4 A B}}$

For $F(z)=0, z^{*}=0$ or $z^{*}=1$ or $z_{1}^{*}=$ $\frac{2 A G}{D+\sqrt{D^{2}-4 A B}}+H$ or $z_{2}^{*}=\frac{2 A G}{D-\sqrt{D^{2}-4 A B}}+H$

And, $A=C_{2}+M_{2}, B=\frac{\left(E_{2}-C_{3}\right) C_{2}\left[\left(1-\mu_{1}\right) P_{1}+V_{3}-\left(1-\mu_{2}\right) P_{2}-V_{1}\right]}{C_{1}\left(V_{2}-V_{1}\right)}$,

$D=\frac{\left.E_{2}-C_{3}\right) C_{2}\left[\left(1-\mu_{1}\right) P_{1}+V_{3}-\left(1-\mu_{2}\right) P_{2}-V_{1}\right]+\left(E_{2}-C_{3}\right) M_{1}\left(V_{2}-V_{1}\right)}{C_{1}\left(V_{2}-V_{1}\right)}$,

$G=\frac{C_{2}}{C_{1}}, F=\frac{\left(1-\mu_{1}\right) P_{1}+V_{3}-\left(1-\mu_{2}\right) P_{2}-V_{1}}{V_{2}-V_{1}}$, 


$$
H=1-\frac{M_{1}}{C_{1}}
$$

In conclusion, it gets ten dynamic balancing points: $\quad O(0,0,0), A(0,0,1), B(0,1,0), C(0,1,1)$, $D(1,0,0), E(1,0,1), F(1,1,0), G(1,1,1), H\left(x_{1}^{*}, y_{1}^{*}, z_{1}^{*}\right)$, $I\left(x_{2}^{*}, y_{2}^{*}, z_{2}^{*}\right)$.The stability of dynamic balancing points can be derivred by analysing the part stability of Jacobian matrix (Friedman 1991). The value of determinant of $J$ is:

$\operatorname{det} J=a_{1} b_{2} c_{3}+a_{2} b_{3} c_{1}+a_{3} b_{1} c_{2}-a_{1} b_{3} c_{2}-$ $a_{2} b_{1} c_{3}-a_{3} b_{2} c_{1}$, The trace value of $J$ is: $\operatorname{tr} J=$ $a_{1}+b_{2}+c_{3}$. If det $J>0$, and $\operatorname{tr} J<0$, dynamic balancing points are in stable state.

According to the value of $H\left(x_{1}^{*}, y_{1}^{*}, z_{1}^{*}\right)$ and $I\left(x_{2}^{*}, y_{2}^{*}, z_{2}^{*}\right), y_{1}^{*}$ is always being negative and $z_{1}^{*}$ is always being passive, which does not affected by the value of $x_{1}^{*}$. Similarly, $y_{2}^{*}$ is always being negative and $z_{2}^{*}$ is always being positive, which does not affected by the value of $x_{2}^{*}$. In this context, only $x^{*}$ will be considered here:

Scenario 1: For $x_{1}^{*}>0, y_{1}^{*}<0, z_{1}^{*}>0, V_{3}-V_{1}<$ $\left(1-\mu_{2}\right) P_{2}-\left(1-\mu_{1}\right) P_{1}$

Scenario 4: For $x_{2}^{*}<0, y_{2}^{*}>0, z_{2}^{*}<0, V_{3}-V_{1}<$ $\left(1-\mu_{2}\right) P_{2}-\left(1-\mu_{1}\right) P_{1}$

In this case, the difference between the treatment effect and cost of patients when they receive treatment provided by specialized hospitals is minor than that of patients when they receive treatment provided by general hospitals without the cooperation with specialized hospitals. The part stability analysis is displayed in Table 1, and dynamics evolution is displayed in Figure 3.

From Table 1 and Figure 3 ,there are 9 partial balancing points: $O(0,0,0), A(0,0,1), B(0,1,0), C(0,1,1)$, $D(1,0,0), E(1,0,1), F(1,1,0), G(1,1,1), H\left(x_{1}^{*}, y_{1}^{*}, z_{1}^{*}\right)$. Among them, $O(0,0,0)$ and $G(1,1,1)$ are in ESS. In this case, $O(0,0,0)$ indicates that patients receive the treatment provided by specialized hospitals and $G(1,1,1)$ indicates that patients received the treatment provided by general hospitals who sent an invitation to specialized hospitals and acquired assistance from them. Plane OAGE is the boundary that distinct each plane. $G(1,1,1)$ is the shrinking point when plane OAGE within HEFG., indicating that patients received the treatment provided by general hospitals who sent an invitation to specialized hospitals and acquired assistance from them.
$O(0,0,0)$ is the shrinking point when plane OAGE within HAOD, indicating that patients received the treatment provided by specialized hospitals.

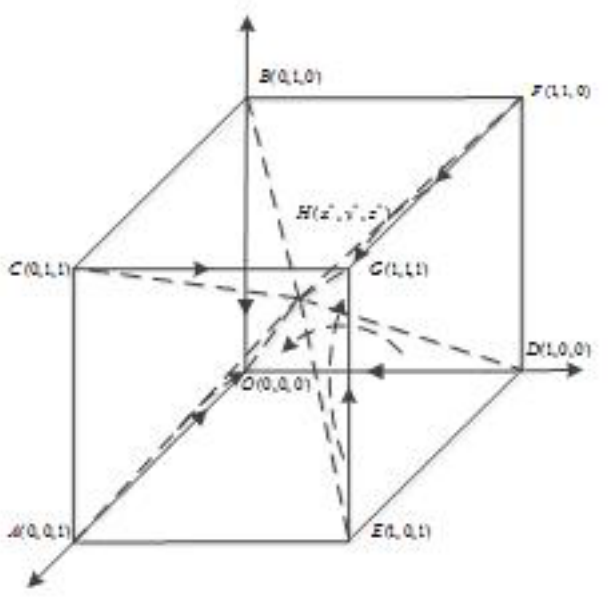

Fig. 3. Dynamics evolutions of system in scenario 1 and 4

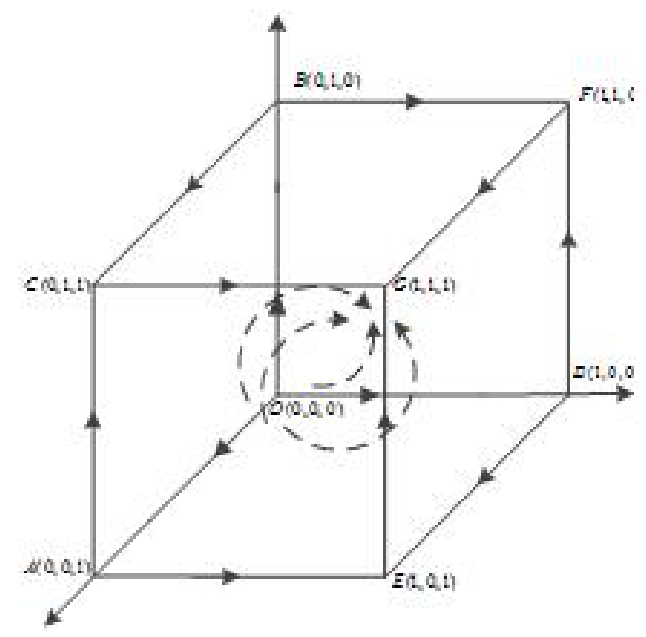

Fig. 4. Dynamics evolutions of system in scenario 2 and 3

Scenario 2: For $x_{1}^{*}<0, y_{1}^{*}<0, z_{1}^{*}>0, V_{3}-V_{1}>$ $\left(1-\mu_{2}\right) P_{2}-\left(1-\mu_{1}\right) P_{1}$

Scenario 3: For $x_{2}^{*}>0, y_{2}^{*}>0, z_{2}^{*}<0, V_{3}-V_{1}>$ $\left(1-\mu_{2}\right) P_{2}-\left(1-\mu_{1}\right) P_{1}$

In this case, the difference between the treatment effect and cost of patients when they receive treatment provided by specialized hospitals is greater than that of patients when they receive treatment provided by general hospitals without the cooperation with specialized hospitals. The part stability analysis is displayed in Table 2, and dynamics evolution is displayed in Figure 4. $G(1,1,1)$ indicates that 
Table 1. Result of analyses of stability in scenario 1 and 4.

\begin{tabular}{|c|c|c|c|c|c|}
\hline Balancing point & $\operatorname{det} \mathrm{J}$ & Symbol & $\operatorname{tr} \mathrm{J}$ & Symbol & Part stability \\
\hline$O(0,0,0)$ & $\begin{array}{c}\left(M_{1}-C_{1}\right)\left(C_{3}-E_{2}\right) \\
{\left[V_{1}\left(1-\mu_{1}\right) P_{1}-V_{3}+\left(1-\mu_{2}\right) P_{2}\right]}\end{array}$ & + & $\begin{array}{c}\left(M_{1}-C_{1}\right)+\left(C_{3}-E_{2}\right)+ \\
{\left[V_{1}\left(1-\mu_{1}\right) P_{1}-V_{3}+\left(1-\mu_{2}\right) P_{2}\right]}\end{array}$ & - & ESS \\
\hline$A(0,0,1)$ & 0 & 0 & $M_{1}-\left[V_{1}-\left(1-\mu_{1}\right) P_{1}-V_{3}+\left(1-\mu_{2}\right) P_{2}\right.$ & - & \\
\hline$B(0,1,0)$ & $\begin{array}{c}\left(M_{1}-C_{1}-C_{2}\right)\left(-C_{3}+E_{2}\right) \\
{\left[V_{1}\left(1-\mu_{1}\right) P_{1}-V_{3}+\left(1-\mu_{2}\right) P_{2}\right]}\end{array}$ & - & $\begin{array}{c}\left(M_{1}-C_{1}-C_{2}\right)+\left(-C_{3}+E_{2}\right)+ \\
{\left[V_{1}\left(1-\mu_{1}\right) P_{1}-V_{3}+\left(1-\mu_{2}\right) P_{2}\right]}\end{array}$ & None & Saddle point \\
\hline$C(0,1,1)$ & 0 & 0 & $\begin{array}{c}\left(M_{1}-C_{2}\right)- \\
{\left[V_{1}\left(1-\mu_{1}\right) P_{1}-V_{3}+\left(1-\mu_{2}\right) P_{2}\right]}\end{array}$ & - & \\
\hline$D(1,0,0)$ & $\begin{array}{c}\left(-M_{1}+C_{1}\right)\left(C_{2}+M_{2}\right) \\
{\left[V_{1}\left(1-\mu_{1}\right) P_{1}-V_{3}+\left(1-\mu_{2}\right) P_{2}\right]}\end{array}$ & + & $\begin{array}{c}\left(-M_{1}+C_{1}\right)+\left(C_{2}+M_{2}\right)+ \\
{\left[V_{1}\left(1-\mu_{1}\right) P_{1}-V_{3}+\left(1-\mu_{2}\right) P_{2}\right]}\end{array}$ & + & Saddle point \\
\hline$E(1,0,1)$ & $-M_{1}\left(C_{2}+M_{2}\right)$ & + & $-M_{1}+\left(C_{2}+M_{2}\right)+$ & None & Saddle point \\
\hline$F(1,1,0)$ & $\begin{array}{c}{\left[V_{1}\left(1-\mu_{1}\right) P_{1}-V_{3}+\left(1-\mu_{2}\right) P_{2}\right]} \\
\left(-M_{1}+C_{1}+C_{2}\right)\left(-C_{2}+M_{2}\right) \\
{\left[V_{1}\left(1-\mu_{1}\right) P_{1}-V_{3}+\left(1-\mu_{2}\right) P_{2}\right]}\end{array}$ & - & $\begin{array}{c}{\left[V_{1}\left(1-\mu_{1}\right) P_{1}-V_{3}+\left(1-\mu_{2}\right) P_{2}\right]} \\
\left(-M_{1}+C_{1}+C_{2}\right)+\left(-C_{2}+M_{2}\right)+ \\
{\left[V_{1}\left(1-\mu_{1}\right) P_{1}-V_{3}+\left(1-\mu_{2}\right) P_{2}\right]}\end{array}$ & None & Saddle point \\
\hline$G(1,1,1)$ & $\begin{array}{c}\left(-M_{1}+C_{2}\right)\left(-C_{2}+M_{2}\right) \\
{\left[V_{1}\left(1-\mu_{1}\right) P_{1}-V_{3}+\left(1-\mu_{2}\right) P_{2}\right]}\end{array}$ & + & $\begin{array}{c}\left(-M_{1}+C_{2}\right)+\left(-C_{2}+M_{2}\right)+ \\
{\left[V_{1}\left(1-\mu_{1}\right) P_{1}-V_{3}+\left(1-\mu_{2}\right) P_{2}\right]}\end{array}$ & - & Ess \\
\hline$H\left(x_{1}^{*}, y_{1}^{*}, z_{1}^{*}\right)$ & $\begin{array}{c}\left(x_{1}^{*}-x_{1}^{* 2}\right)\left(y_{1}^{*}-y_{1}^{* 2}\right)\left(z_{1}^{*}-z_{1}^{* 2}\right)\left(V_{2}-V_{1}\right) \\
{\left[\left(E_{2}-C_{3}\right)\left(x_{1} c_{1}^{*}-x_{1}^{*} z_{1}^{*} C_{1}-y_{1}^{*} C_{2}+\right.\right.} \\
\left.\left.x_{1}^{*} y_{1}^{*} C_{2}\right)+x_{1}^{*} C_{1}\left(M_{2}+C_{2}\right)\right]\end{array}$ & - & 0 & & Saddle point \\
\hline$I\left(x_{2}^{*}, y_{2}^{*}, z_{2}^{*}\right)$ & $\begin{array}{c}\left(x_{2}^{*}-x_{2}^{* 2}\right)\left(y_{2}^{*}-y_{2}^{* 2}\right)\left(z_{2}^{*}-z_{2}^{* 2}\right)\left(V_{2}-V_{1}\right) \\
{\left[\left(E_{2}-C_{3}\right)\left(x_{2} c_{1}^{*}-x_{2}^{*} z_{2}^{*} C_{1}-y_{2}^{*} C_{2}+\right.\right.} \\
\left.\left.x_{2}^{*} y_{2}^{*} C_{2}\right)+x_{2}^{*} C_{1}\left(M_{2}+C_{2}\right)\right]\end{array}$ & - & 0 & & Saddle point \\
\hline
\end{tabular}

Table 2. Result of analyses of stability in scenario 2 and 3.

\begin{tabular}{cccccccc}
\hline Balancing point & Symbol & Symbol & Part stability & Balancing point & Symbol & Symbol & Part stability \\
\hline$O(0,0,0)$ & - & - & saddle point & $D(1,0,0)$ & - & None & Saddle point \\
$A(0,0,1)$ & 0 & + & saddle point & $E(1,0,1)$ & - & None & Saddle point \\
$B(0,1,0)$ & + & None & saddle point & $F(1,1,0)$ & + & None & Saddle point \\
$C(0,1,1)$ & & + & saddle point & $G(1,1,1)$ & + & - & ESS \\
$H\left(x_{1}^{*}, y_{1}^{*}, z_{1}^{*}\right)$ & - & & saddle point & $I\left(x_{2}^{*}, y_{2}^{*}, z_{2}^{*}\right)$ & - & & Saddle point \\
\hline
\end{tabular}


patients received the treatment provided by general hospitals who sent an invitation to specialized hospitals and acquired assistance from them. To sum up, only when $V_{3}-V_{1}<\left(1-\mu_{2}\right) P_{2}-\left(1-\mu_{1}\right) P_{1}$, can the difference between the treatment effect and cost of patients when they receive treatment provided by specialized hospitals be minor than that of patients when they receive treatment provided by general hospitals without the cooperation from specialized hospitals. Only in this case, can the returns among general hospitals, specialized hospitals and patients correlated with each party. This paper analyzes the game theory of trans-regional hospitals and patients under this condition (Liu and Ai 2015).

\section{A case study}

The above-mentioned game model shows that there are two evolutionary results of the behaviors of hospitals and patients in the Telemedicine System: patients choose general hospitals in the cooperation between hospitals; and patients choose specialized hospitals when general hospitals do not cooperate with specialized hospitals. The final equilibrium point of the evolutionary system depends on the game pay-off matrix, the initial value of the parameters and its variations. The influence of the variations of several parameters on system evolutionary results is discussed in this section and Matlab is used to make a numerical analysis on some key parameters. Graphics is used to analyze visually the impact of different states on the evolutionary results of the three sides' choices.

In order to make the study more realistic, the data in the China Health and Family Planning Statistical Yearbook 2015, combined with the data published by the relevant local authorities, is selected and summarized as the initial data, as is shown in Figure 4. In Beijing and Zunyi City in Guizhou Province, for example, hospital costs in Zunyi City are 5176 yuan per capita, municipal hospital reimbursement proportion is $75 \%$, and remote reimbursement proportion is $65 \%$; while the remote consultation fees in Beijing are 2,000 yuan per person and the hospital costs are 18,786 yuan per capita. Assuming that the utility in a specialized hospital is 1 and the utility in a general hospital is 0.2 , a patient can get 0.9 of the utility in a general hospital after the Telemedicine System. Table 5 shows the initial values of the parameters.

According to the description of Section 2, we summarized all assumptions in table 4 . We assume that $x(0 \leqslant x \leqslant 1)$ is represented for the probability for general hospitals sending an invitation to specialized hospitals, $y(0 \leqslant y \leqslant 1)$ is represented for the probability for specialized hospitals accepting an invitation from general hospitals, $z(0 \leqslant z \leqslant 1)$ is represented for the probability for patients to take treatments at general hospitals, $E_{1}$ is represented for specifies clinical cost of general hospitals, $E_{2}$ is represented for specifies clinical cost of specialized hospitals, $M_{1}$ is represented for specifies government fund for remote cooperative medical services, $M_{2}$ is represented for specifies government fund for remote cooperative medical services, $C_{1}$ is represented for specifies operation cost of general hospitals, $C_{2}$ is represented for specifies general hospitals cooperation cost with specialized hospitals, $C_{3}$ is represented for specifies operation cost of specialized hospitals, $P_{1}$ is represented for specifies the cost of patients if they accept medical services at general hospitals, $P_{2}$ is represented for specifies the cost of patients if they accept medical services at specialized hospitals, $\mu_{1}\left(0<\mu_{1}<1\right)$ is represented for specifies the radio of medical insurance of general hospitals, $\mu_{2}\left(0<\mu_{2}<1\right)$ is represented for specifies the radio of medical insurance of specialized hospitals, $V_{1}$ is represented for specifies the medical services effects of patients in general hospitals when specialized hospitals lack cooperation with general hospitals, $V_{2}$ is represented for specifies the medical services effects of patients in general hospitals when specialized hospitals cooperate with general hospitals, $V_{3}$ is represented for specifies the medical services effects of patients in specialized hospitals.

(1) Impact of the initial proportion $(x, y, z)$ of the three sides on the system evolutionary results.

The results of numerical analysis in Figures 5, 6 and 7 show the evolutionary trajectory of patients, general hospitals and specialized hospitals strategy selection behaviors. The contrast of the subgraphs in Figures 5, 6 and 7 demonstrates: whether gen- 
Table 3. Comparison of medical data in Beijing and Guizhou hospitals (CNY).

\begin{tabular}{ccc}
\hline & Beijing & Guizhou \\
\hline Number of Medical Institutions & 9638 & 28995 \\
Medical Income & $122,907,070,000$ & $37,928,160,000$ \\
Medical Expenditure & $116,289,070,000$ & $34,037,970,000$ \\
Average Income of Medical Institutions & $12,750,000$ & $1,310,000$ \\
Average Expenditure of Medical Institutions & $12,070,000$ & $1,174,000$ \\
Hospital Cost per capita & 18,800 & 5,200 \\
Fiscal Subsidy & $22,543,940,000$ & $7,734,180,000$ \\
Average Fiscal Subsidy & $2,339,000$ & 267,000
\end{tabular}

PS: It is assumed that the specialized hospital is in Beijing and the general hospital is in Guizhou.

Table 4. Initial values of the parameters.

\begin{tabular}{ccccccccc}
\hline $\mathrm{x}$ & $\mathrm{y}$ & $\mathrm{z}$ & $E_{1}$ & $E_{2}$ & $M_{1}$ & $M_{2}$ & $C_{1}$ & $C_{2}$ \\
\hline 0.5 & 0.5 & 0.5 & 131 & 1275 & 27 & 224 & 117 & 0.2 \\
\hline$C_{3}$ & $P_{1}$ & $P_{2}$ & $m u_{1}$ & $m u_{2}$ & $V_{1}$ & $V_{2}$ & $V_{3}$ & \\
\hline 1207 & 0.5 & 2 & 0.75 & 0.65 & 0.2 & 0.8 & 1 & \\
\hline
\end{tabular}

eral hospitals send invitations for collaborative medical services to specialized hospitals and whether specialized hospitals accept the invitations depends largely on the initial proportion of patients strategy selection; while the initial proportion of general and specialized hospitals strategy selection makes little differences on patients choice. This shows another important factor affects patients choice.

(2) Impact of different reimbursement proportions of $\mu_{1}$ and $\mu_{2}$ for medical insurance of patients on the system evolutionary results.

The results of numerical analysis are shown in Figures 8 and 9. Figure 8 shows that the strategy selection of general and specialized hospitals evolves in conformity in the uncollaborative direction, and the patient groups evolve towards the choice of specialized hospitals regardless the variations of $\mu_{1}$. The smaller the reimbursement proportion of general hospitals is, the faster the strategy selection of patient groups converge. This shows that the medical insurance reimbursement proportion of general hospitals does not influence the strategy selection of general and specialized hospitals, and does not play a decisive role in affecting patients choices.

Figure 9 indicates that as the medical insurance reimbursement proportion $\mu_{2}$ of specialized hospi- tals decreases, general hospitals move to initiate the invitation of collaborative medical services, the specialized groups evolve to accept the invitation, and the patient groups tend to go to general hospitals for treatment. The smaller $\mu_{2}$ is, the faster the strategy selection of general hospitals, specialized hospitals and the patient groups converges to the equilibrium state. This shows that reducing the medical insurance reimbursement proportion of specialized hospitals can improve the development of Telemedicine System.

(3) Impact of government funding $M_{1}$ and $M_{2}$ on the evolution results.

The results of numerical analysis of the impact of government funding $\left(M_{1}\right.$ and $\left.M_{2}\right)$ on the evolution are shown in Figures 10 and 11. Figure 10 indicates that as $M_{1}$ increases when it starts from $M_{1}=55$, general hospitals tend to initiate the invitation of collaborative medical services, specialized hospitals evolve to accept the invitation, and the patient groups evolve towards general hospitals. As government funding for general hospitals increases, the time for the strategy selection of general hospitals, specialized hospitals and the patient groups to converge to the equilibrium state gradually decreases. This shows that government support for 
general hospitals plays a positive role in promoting the development of Telemedicine System.

Figure 11 shows that general hospitals finally evolve to not initiate the invitation of collaborative medical services regardless of the variations of $M_{2}$; patient groups evolve towards the choice of specialized hospitals for treatment. As government funding for specialized hospitals increases, specialized hospitals first consider to accept the invitation, but soon move towards the refusal of the invitation. This indicates that government support for specialized hospitals has nothing to do with the development of collaborative telemedicine and even hinders its development.

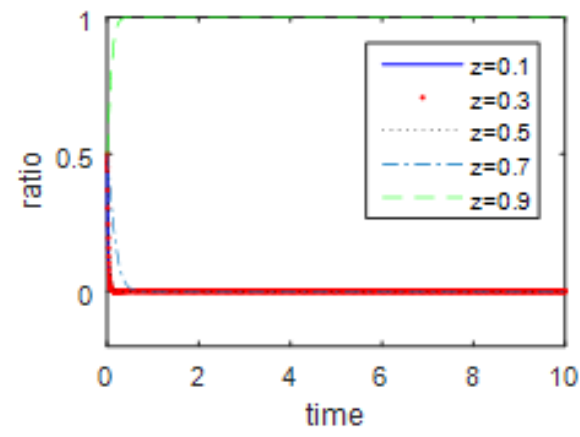

(a) $x=0.5, y=0.5$

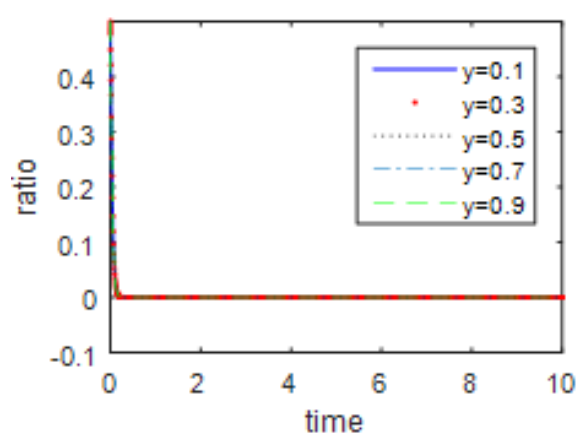

(b) $x=0.5, z=0.5$

Fig. 5. Influence of different initial proportion of game groups on the evolution of general hospitals

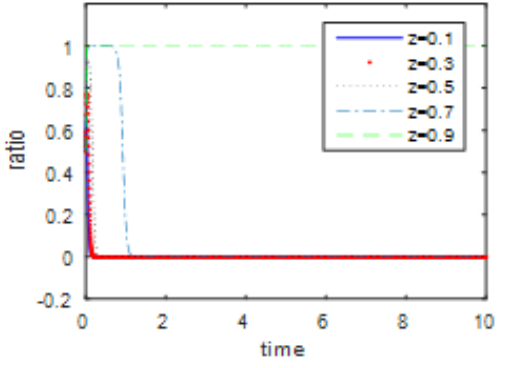

(a) $x=0.5, y=0.5$

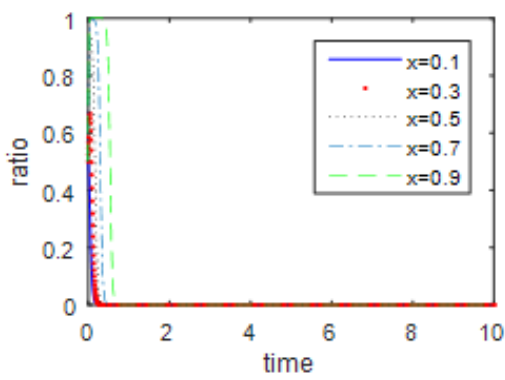

(b) $y=0.5, z=0.5$

Fig. 6. Influence of different initial proportion of game groups on the evolution of specialized hospitals 


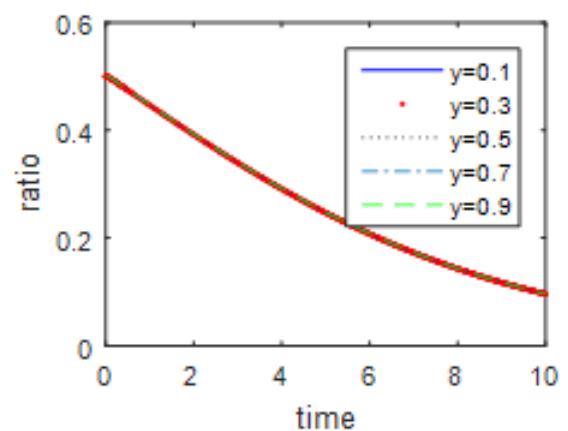

(a) $x=0.5, z=0.5$

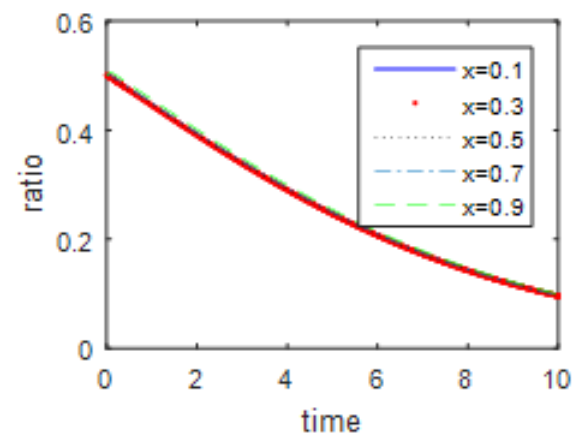

(b) $y=0.5, z=0.5$

Fig. 7. Influence of different initial proportion of game groups on the evolution of patients

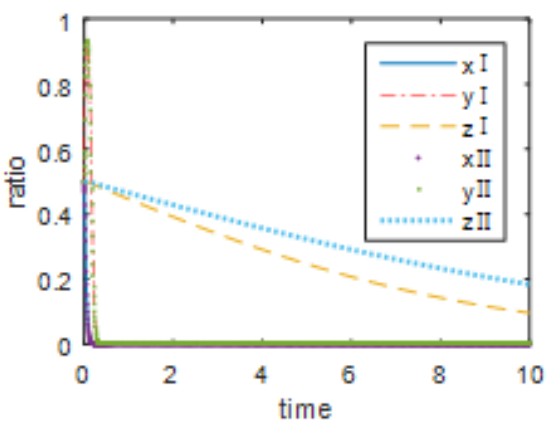

(a) $\mu 1=0.9$

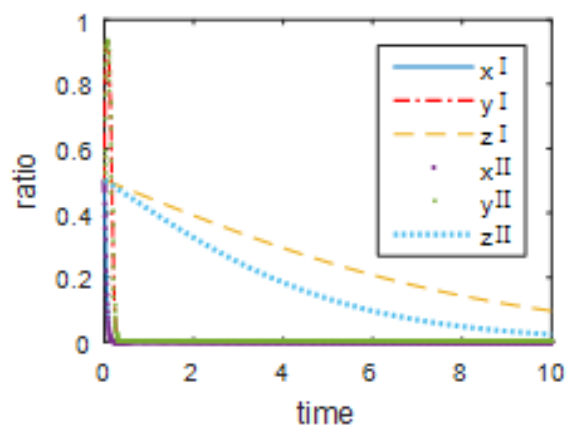

(b) $\mu 1=0.3$

Fig. 8. Impact of medical insurance reimbursement proportion $\mu_{1}$ of general hospitals on evolution

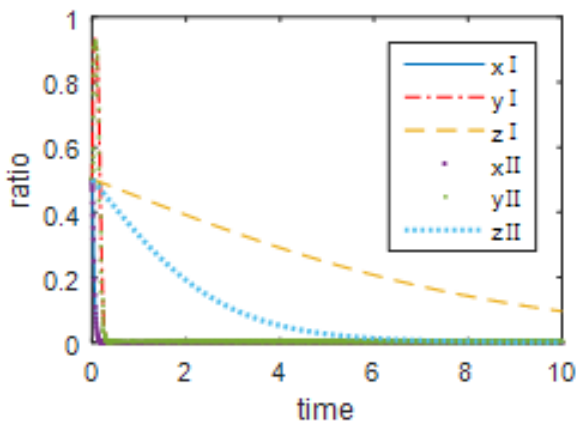

(a) $\mu 2=0.9$

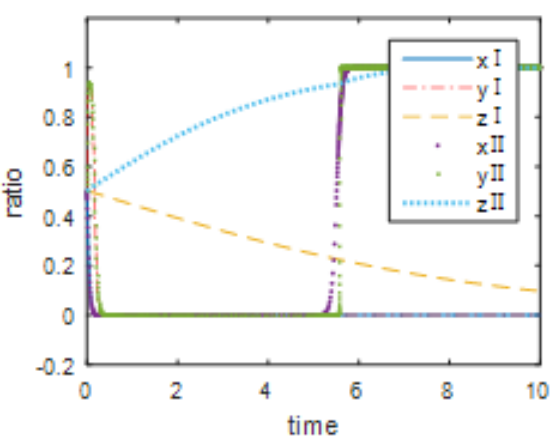

(b) $\mu_{2}=0.3$

Fig. 9. Impact of medical insurance reimbursement proportion $\mu_{2}$ of specilaized hospitals on evolution 


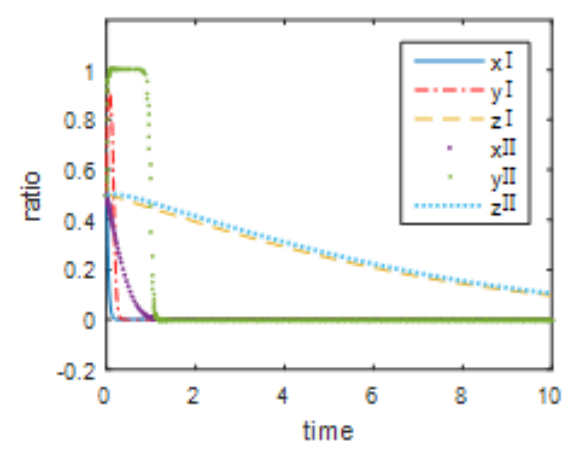

(a) $M_{1}=55$

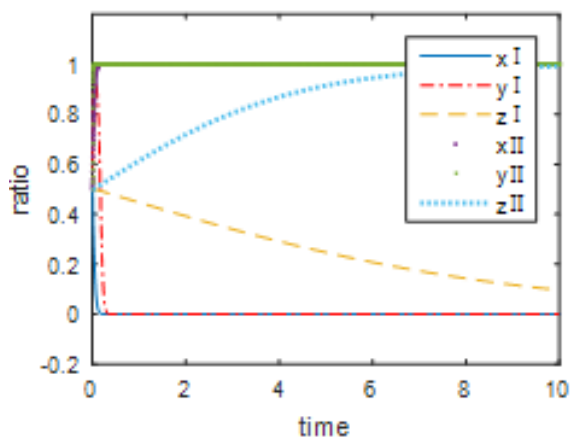

(b) $M_{1}=60$

Fig. 10. Impact of government funding for general hospitals $M_{1}$ on evolution

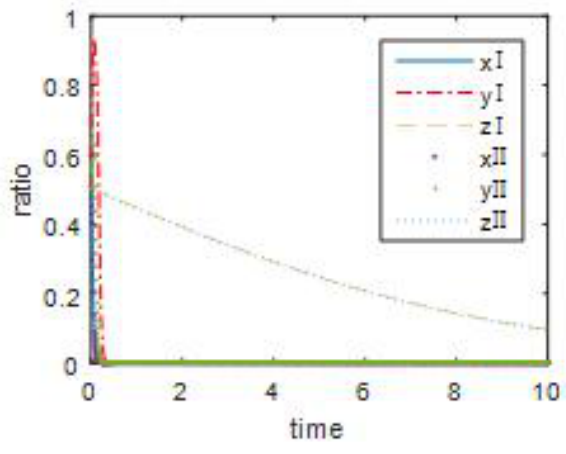

(a) $M_{2}=100$

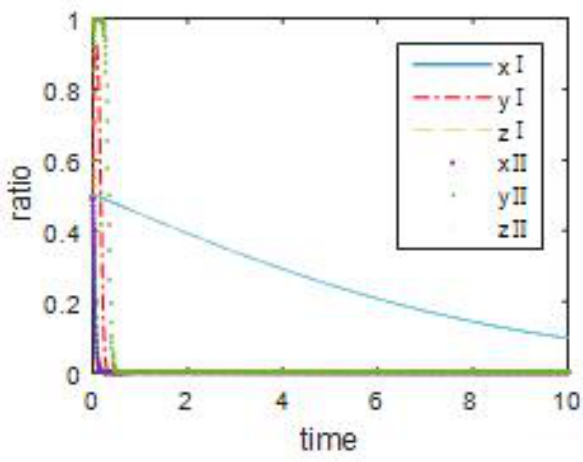

(b) $M_{2}=500$

Fig. 11. Impact of government funding for specialized hospitals $M_{2}$ on evolution

(4) Impact of the payment of patients in different hospitals $P_{1}$ and $P_{2}$ on the system evolutionary results.

The results of numerical analysis are shown in Figures 12 and 13. Figure 12 shows that general hospitals evolve to not initiate the invitation of collaborative medical services regardless of the variations of $P_{1}$; specialized hospitals first choose to accept the collaborative invitation but soon move to refuse the invitation; and patients tend to choose specialized hospitals for treatment. As the payment of patients in general hospitals $P_{1}$ increases, the time for patients to converge to the equilibrium state becomes shorter. This shows the strategy selection of general hospitals and specialized hospitals is free from the variations of $P_{1}$ and does not play a decisive role in affecting patients choices. 


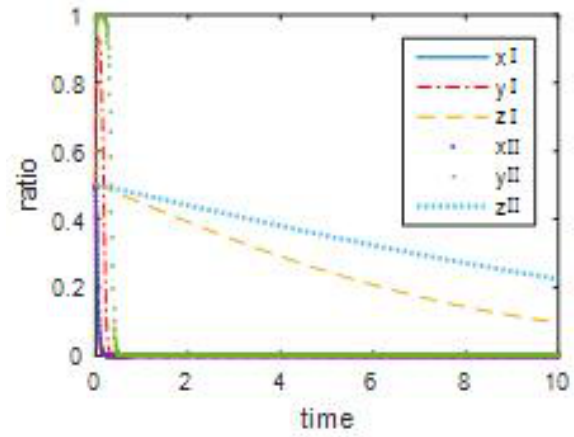

(a) $P_{1}=0.1$

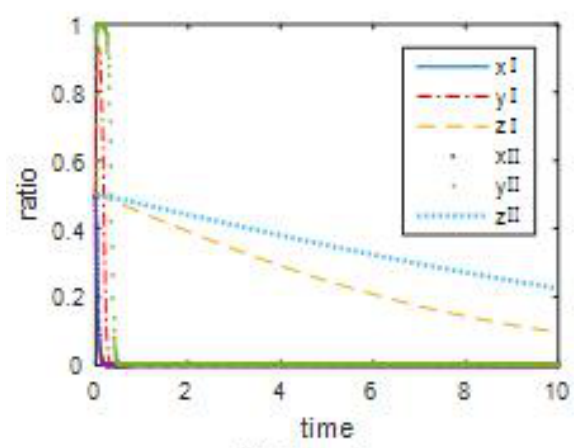

(b) $P_{1}=2$

Fig. 12. Impact of the payment in general hospitals $P_{1}$ on evolutionary results

Figure 13 shows that as the payment of patients in specialized hospitals $P_{2}$ increases, patient groups evolve towards the choice of general hospitals, and the time to converge to the equilibrium state gradually reduces. When $P_{2}=3$, general hospitals evolve to not invite collaborative medical services. When $P_{2}=6$, general hospitals eventually evolve in the direction of initiating the invitation after a short period when they first evolve to not initiate an invitation. As the value of $P_{2}$ increases, the time to converge to the equilibrium state gets shorter. Specialized hospitals initially move to accept the invitation, but soon evolve to refuse it. When the value of $P_{2}$ reaches a certain value, specialized hospitals tend to accept the invitation. As the value of $P_{2}$ increases, the time for specialized hospitals to converge to the equilibrium state becomes shorter. This shows that increasing the cost of diagnosis and treatment of specialized hospitals plays a positive role in the implementation of Telemedicine Systems.

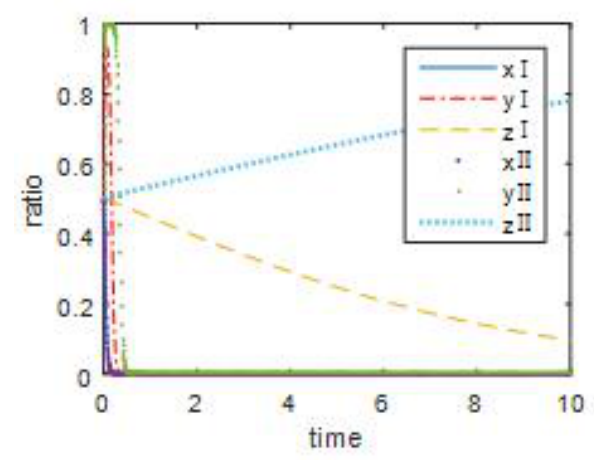

(a) $P_{2}=3$

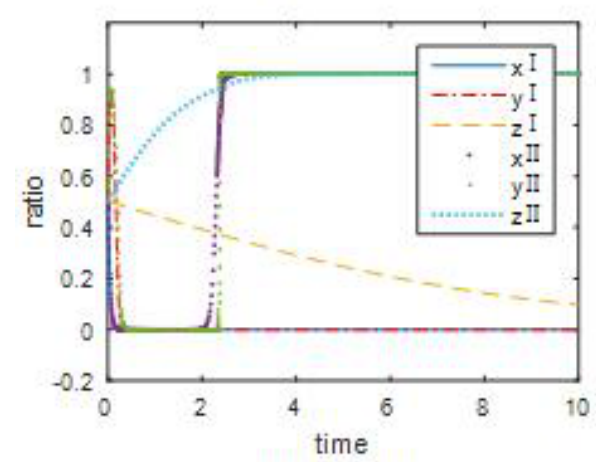

(b) $P_{2}=6$

Fig. 13. Impact of the payment in specialized hospitals $P_{2}$ on evolutionary results

(5) Impact of different operation costs $C_{1}$ and $C_{3}$ on evolutionary results

The results of numerical analysis are shown in Figures 14 and 15. Figure 14 shows that as the operation costs of the general hospitals decrease, general hospitals, specialized hospitals and patient groups evolve towards the choice of collaborative medical services. When $C_{1}=60$, general hospitals evolve to not invite collaborative medical services. Specialized hospitals eventually evolve in the direction of not accepting the invitation after a short period when they first evolve to accept an invitation. Patient groups evolve towards the choice of specialized hospitals. When $C_{1}=55$, general hospitals choose to invite the collaborative invitation, specialized hospitals choose to accept the collaborative invitation, patient groups evolve towards the choice of general hospitals. As the value of $C_{1}$ decreases, the time to converge to the equilibrium state becomes shorter. 


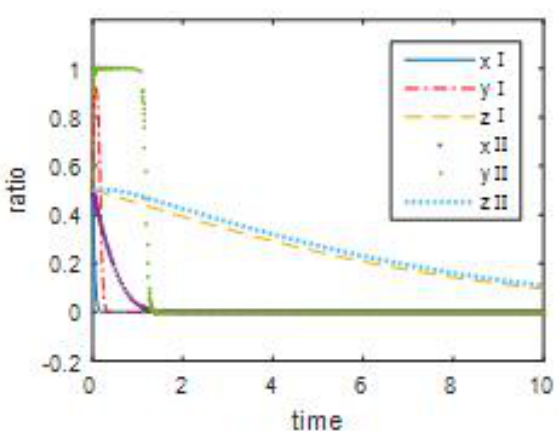

(a) $C_{1}=60$

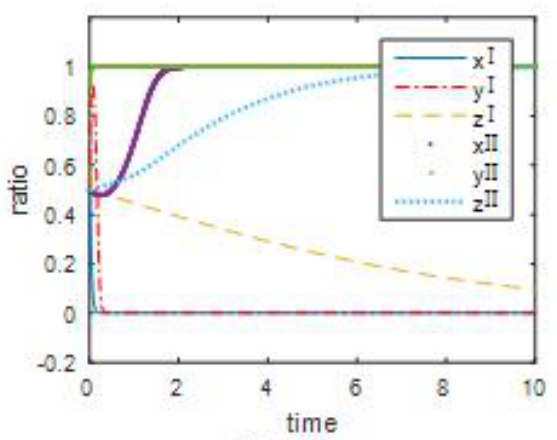

(b) $C_{1}=55$

Fig. 14. Impact of operation costs of general hospitals $C_{1}$ on evolution

Figure 15 shows that general hospitals evolves in the direction of not initiating collaborative medical service invitations independent of the value of $C_{3}$; special hospitals initially choose to accept the invitations but soon does not accept; More patients choose special hospital for treatment. This indicates that the strategies chosen by general hospitals, special hospitals and patients are not affected by the value of $C_{3}$. This shows that reducing the operating costs of general hospitals helps promote the development of Telemedicine Systems.

Finally, the parameters that have a greater impact on the final outcome are adjusted together to achieve the desired ideal state as shown in Figure 16, in which general hospitals evolves in the direction of not initiating collaborative medical service invitation; Special hospitals are approached to accept the invitation; More patients choose general hospitals for treatment. The values of the three variables are as follows: $\mu_{2}=0.3, M_{1}=60, P_{2}=6, C_{1}=55$.

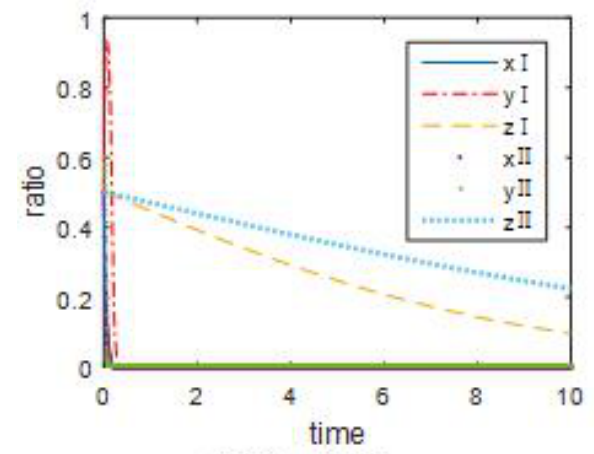

(a) $C_{3}=1000$

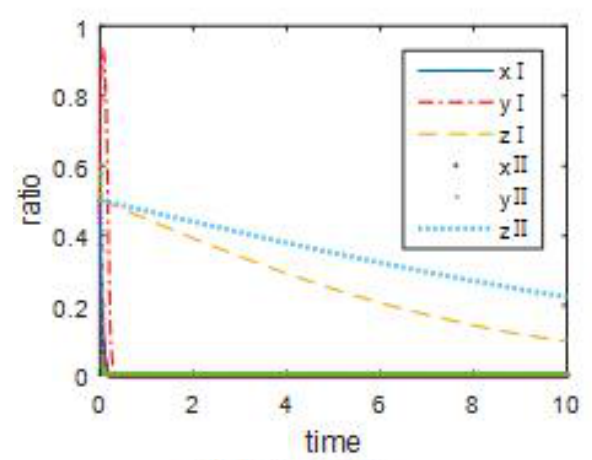

(b) $C_{3}=500$

Fig. 15. Impact of operation costs of specialized hospitals $C_{1}$ on evolution

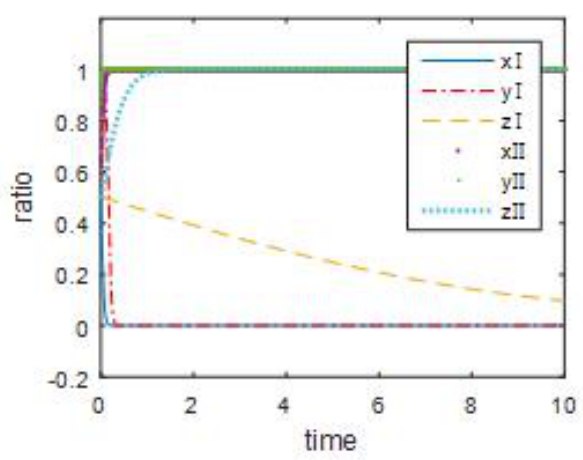

Fig. 16. Impact of $\mu_{2}, M_{1}$ and $P_{2}$ on evolution

\section{Conclusion and further remarks}

The telemedicine system is so comprehensive and time-consuming that deserves the consistent investment, reform and further improvement from our government. Whether general hospitals, specialized hospitals and patients choose remote cooperative medical services or not depends on variable condi- 
tions, such as times, cultural backgrounds and economy. Moreover, existing studies fail to provide practical models that influence and restrict the development of remote cooperative medical services. This paper establishes evolutionary game model about the influences on selection behavior of general hospitals, specialized hospitals and patients by analyzing statistic from the China Health and Family Planning Statistical Yearbook 2015 and emulating calculation. This paper offers not only a new way of thinking but an innovative breakthrough for evolutionary game method finding its place in the field of telemedicine.

This paper concludes that to promote the development of telemedicine, we need to decrease the proportion of current medical insurance at special hospitals, to increase government funding for general hospitals and treatment charges at special hospitals as well as to reduce the operating cost of special hospitals. In order to encourage general hospitals to send invitations to special hospitals and encourage patients to accept telemedicine services at local general hospitals, the following recommendations were provided:

(1) Medical insurance reimbursement rates in special hospitals charge influence on patients' choices, as over reimbursement attracts almost all patients to have treatments in special hospitals. Therefore, the government is advised to set up reasonable standard of reimbursement rate in special hospitals by use of policy leverage of the medical insurance, guiding patients to have treatments in general hospitals for those diseases that can be cured by services of the remote cooperative medical system.

(2) Our presumption informs that, general hospitals are usually located in relatively remote and under-developed provinces and cities where the information exchanges are insufficient, therefore these hospitals lack depth recognition to the remote cooperative medical services. In addition, government funding for development of these hospitals are relatively small. So it is inferred that general hospitals are not motivated enough to promote the development of the remote cooperative medial services. So it is advised that the government enhance financial support for general hospitals when budgeting the fu- ture health expenditure. Beside the financial support, policies and related technical assistance also should be provided by the government.

(3) Due to the rise of people's living standard, patients have switched primary concern from treatment charges to treatment effects. As a result, patients prefer to visit special hospitals if it is financially available for them. As it stands, concerning the promotion of remote cooperative medical services, special hospitals are advised to properly increase prices of medical services, so as to disperse patients into a more reasonable distribution and improve the graded medical constitution.

(4) It is typical for general hospitals to have unwieldy organizations with heavy amount of departments and locate in remote and under-developed regions. Consequently, they are facing problems of high operating costs and low overall profits which baffles the wide practice of remote cooperative medical services. And so, general hospitals are advised to implement countermeasures such as cost accounting, internal management enhancement and internal mechanism improvement to finally reduce operating cost and maximize the efficiency of their medical services.

(5) In addition to factors analyzed above, it is advised that government adopt more measures to boost medical service quality of general hospitals, strengthen their market competitiveness, encourage patients to make more wise decisions on hospital choosing, balance burdens among different medical services, and advance services efficiency.

In building and developing the remote cooperative medical services, it is required the coordination and interactions among the three parties of general hospitals, special hospitals and patients. Parameters in the study of this paper are set up based on an extensive selection of factors influencing the three parties. However, psychological factors of patients and particularities of regional hospitals are missed in this study. In future work, more efforts will be made to analyze other problems in the system of remote cooperative medical services, thus accelerating the development of this beneficial business. 


\section{Acknowledgments}

This work was supported by National Natural Science Funds of China (Nos.71571090, 71161016, 71401131 and 61563029), the Soft Science Funded Project of Shaanxi Province (Nos. 2017KRM192 and 2017KRM005), the Natural Science Foundation Research Project of Shaanxi Province (No. 2017JM7022), the Social Science Planning Project Fund of Xi' an (17J64) and the Key Strategic Project of Fundamental Research Funds for the Central Universities (JBZ170601).

\section{References}

1. S. Z. Ai and X. N. Fan,Evolutionary Game Analysis of Choice Behaviors to Medical APP by Hospitals and Patients Chinese J Manage Sci,23 (11) (2015) 34-40.

2. Q. X. An, F. Y. Meng, S. Ang and X. H. Chen, A new approach for fair efficiency decomposition in twostage structure system, Oper Res,(2016) 1-16.

3. D. Friedman, Evolutionary games in economics. Econometric.Journal of the Econometric Society 59 (3): 637-666

4. Z. Fu, Telemedicine. Beijing: Peoples Military Medical Press,2006, pp.6-7.

5. Z.J. Fu, K. Ren, J.G.Shu, X.M. Sun, F.X. Huang. Enabling personalized search over encrypted outsourced data with efficiency improvement. IEEE T Parall Distr 27 (9) (2017) 2546-2559.

6. Y.Q. Gao, M.X. You, K. Hu. Research on relations among medical insurance reimbursement disparity, patients behaviors and medical expenses-analysis based on three-phase dynamic game theory model. Syst Eng Theor Pract 34 (11): 2974-2983.

7. B.Gu, V.S. Sheng. A robust regularization path algorithm for -support vector classification. IEEE T Neur Net Lear, 99 (2015) 1-8.

8. B.Gu, V.S. Sheng, Z.J. Wang, D.H.S. Osman, S. Li. Incremental learning for -support vector regression. Neural Networks, 67 (2015) 140-150.

9. M.Z. Jia, X. Lei, J. Du. Evolutionary Game Theory in Multi-Objective Optimization Problem. Int J Comput Int Sys 12 (1) (2010) 74-87.

10. Y. Kong, M.J. Zhang, D.Y. Ye. A belief propagationbased method for task allocation in open and dynamic cloud environments. Knowl-Based Syst, 115 (2016) 113-132.

11. D.F. Li. An effective methodology for solving matrix games with fuzzy payoffs. IEEE T Cyebernetics 43 (2) (2013) 610-621.

12. D.F. Li, J.C. Liu. A parameterized non-linear pro- gramming approach to solve matrix games with payoffs of I-fuzzy numbers. IEEE T Fuzzy Syst 23 (4) (2015a) 885-896.

13. D.F. Li, J. Yang. A parameterized non-linear programming approach to solve matrix games with payoffs of I-fuzzy numbers. IEEE T Fuzzy Syst 23 (4) (2015b) $885-896$.

14. D.F. Li. A parameterized non-linear programming approach to solve matrix games with payoffs of I-fuzzy numbers. IEEE T Fuzzy Syst 23 (4) (2014) 885-896.

15. J. Li, X.L. Li, B. Yang, X.M. Sun. Segmentationbased image copy-move forgery detection scheme. IEEE T Inf Forem Sec 10 (3) (2015) 507-518.

16. F.Y. Meng, Q. Zhang, Y. Wang. Cooperative fuzzy games with a coalition structure and interval payoffs. IEEE T Fuzzy Syst 20 548-558.

17. F.Y. Meng. Cooperative fuzzy games with convex combination form. Asia Pac J Oper Res 23 (4) (2015) 885-896.

18. F.Y. Meng, Q. Zhang, X.H. Chen. Fuzzy multichoice games with fuzzy characteristic functions. Group Decis Nego 23 (4) (2016) 885-896.

19. J.X. Nan, D.F. Li. Linear programming approach to matrix games with intuitionistic fuzzy goals. Int $J$ Comput Int Sys 6 (1) (2013) 186-197.

20. B. Rajan, A. Seidmann, E.R. Dorsey. The competitive businessimpact of using telemedicine for the treatment of patients with chronic conditions. $J$ Manage Inform Syst 30 (2) (2013) 127-158.

21. B.O. Sarah, H. Slim. A multi-criteria optimization approach to health care tasks scheduling under resources constraints. Int J Comput Int Sys 10 (2017) 419-439.

22. B.Z. Sun, W.M. Ma. Rough approximation of a preference relation by multi-decision dominance for a multiagent conflict analysis problem. Inform Sciences $\mathbf{3 1 5}$ (2015a) 39-53.

23. B.Z. Sun, W.M. Ma. An approach to consensus measurement of linguistic preference relations in multiattribute group decision making and application. Omega 51 (2015b) 83-92.

24. B.Z. Sun, W.M. Ma, H.Y. Zhao. Rough set-based conflict analysis model and method over two universes. Inform Sciences 372 (2016a) 111-125.

25. B.Z. Sun, W.M. Ma, H.Y. Zhao. An approach to emergency decision-making based on decision-theoretic rough set over two universes. Soft Comput 20 (9) (2016b) 3617-3628.

26. B.Z. Sun, W.M. Ma, X. Xiao. Three-way group decision making based on multigranulation fuzzy decision-theoretic rough set over two universes. Int $J$ Approx Reason 81 (2017) 87-102.

27. V. Tina, K. Amit. A Note on A lexicographic method for matrix games with payoffs of triangular intuitionistic fuzzy numbers. Int J Comput Int Sys 4 (8) (2017) 690-700. 
28. S.B. Wang. Reflections on the application of telemedicine in China. China Medical Education Technology 12 (2012) 676-679.

29. S.X. Wang, R. Du, S.Z. Ai, Z.M. Zheng. The evolution analysis of community hospitals and patients behavior selection under background of telemedicine. Indust Eng and Manage 2 (20) (2015) 130-137.

30. X.Z. Wen, L. Shao, Y. Xue, W. Fang. A rapid learning algorithm for vehicle classification. Inform Sciences $\mathbf{1}$ (295) (2015) 395-406.

31. Z.H. Xia, X.H. Wang, X.M. Sun, Q.S. Liu, N.X.Xiong. Steganalysis of LSB matching using differences between nonadjacent pixels. Multimed Tools Appl, 75 (4) (2016) 1947-1962.

32. D.Y. Xie, F. Guan, Q. Zhang. A further research on score for interval cooperative games. Int J Comput Int Sys 2 (8) (2015) 307-316.

33. F.L. Ye, G.L. Shi. Research on telemedicine and its collaboration. Information of Medical Equipment $\mathbf{8}$ (2006) 32-34.

34. C.S.Yuan, X.M. Sun, R.Lv. Fingerprint liveness detec- tion based on multi-scale LPQ and PCA. China Commun, 13 (7) (2016) 60-65.

35. P. Zanaboni, R. Wootton. Adoption of telemedicine: from pilot stage to routine delivery. BMC Med Inform Decis 12 (1) (2012) 1-9.

36. J.J. Zheng, W.L. Jiang, Z.X. Zou, X. Han. Research on bidding equilibrium of $\mathrm{VC}$ exit discriminatory auction system based on evolutionary game. Chinese J Manage Sci 2 (1) (2013) 185-192.

37. Y.H. Zheng, B.W. Jeon, D.H. Xu, Q.M.J. Wu, H. Zhang. Image segmentation by generalized hierarchical fuzzy C-means algorithm. J Int Fuzzy Syst 2 (28) (2015) 961-973.

38. Z.L. Zhou, Y.L. Wang, Q.M.J. Wu, C.N. Yang, X.M. Sun. Effective and efficient global context verification for image copy detection. J Int Fuzzy Syst 12 (1) (2017) 48-63.

39. Z.L. Zhou, C.N. Yang, B.J. Chen, X.M. Sun, Q. Liu, Q.M.J. Wu. Effective and efficient image copy detection with resistance to arbitrary rotation. Multimed Tools Appl, 99 (6) (2016) 1531-1540. 